\title{
Static Electric-Spring and Nonlinear Oscillations
}

\author{
Haiduke Sarafian
}

University College, The Pennsylvania State University, York, USA.

Email: has2@psu.edu

Received October $13^{\text {th }}, 2009$; revised November $18^{\text {th }}, 2009$; accepted November $25^{\text {th }}, 2009$.

\begin{abstract}
The author designed a family of nonlinear static electric-springs. The nonlinear oscillations of a massively charged particle under the influence of one such spring are studied. The equation of motion of the spring-mass system is highly nonlinear. Utilizing Mathematica [1] the equation of motion is solved numerically. The kinematics of the particle namely, its position, velocity and acceleration as a function of time, are displayed in three separate phase diagrams. Energy of the oscillator is analyzed. The nonlinear motion of the charged particle is set into an actual three-dimensional setting and animated for a comprehensive understanding.
\end{abstract}

Keywords: Static Electric-Spring, Nonlinear Oscillator, Mathematica

\section{Introduction}

Analysis of the kinematics and dynamics of a mechanical spring-mass system is a classic physics problem [2]. In this analysis the spring is idealized; it is assumed the spring is mass-less and linear. The first assumption is "justified" when the mass of the object outweighs the spring. The linearity for a coiled-shaped spring for most of the time is enforced by not stretching the spring beyond its plastic limit. Under these assumptions the equation describing the motion of the object is a second order linear differential equation; it is trivially solved with analytic sinusoidal solutions. This scenario is modified slightly when a nonlinear mass-less spring is considered. For instance, some 90 years ago, Duffing [3] initiated the notion of the nonlinear vibrator. Accordingly, an equation is proposed to describe the state of one such oscillator. Duffing's equation is given by, $\ddot{x}+\alpha \dot{x}+\beta x+\gamma x^{3}=f(t)$; where, in addition to the linear spring term, $\beta x$, a cubic term, $\gamma x^{3}$, is used to characterize the non-linearity. Damping and the source of the forced oscillation terms are given by, $\alpha \dot{x}$, and $f(t)$, respectively. The notion of Duffing's initiated nonlinear vibrator has found its applications in a wide range of scientific fields. Reference [4] for instance has sections devoted to the description of the Duffing equation. The latter reference also contains a wealth of bibliographic listed related articles. Recently, an electronic website [5] posted an animated description of the Duffing related issues. However, neither these references nor the author's thorough literature search could identify a source describing the oscillations of an ideall perfect mass-less mechanical vibrator. The article that "best" aligns with one such mechanical oscillation is a suggested experiment given in [6]. The authors of the latter reference have claimed their proposed experiment would produce data that is compatible with the description of the Duffing equation. However, a careful analysis of their setup and suggested analysis reveals the mass of the elastic metal strip is ignored. This leads to expect disconnect between the data and the proposed theory.

Motivated with identifying the missing practical design of mechanical oscillations of an ideal/perfect mass-less oscillator, the author designed a spring made of a static electric field, and called it an electric-spring. The point is a spring made of an electric field is a mass-less spring. In other words, there is no need to make assumptions justifying the smallness of the spring's mass. This makes the spring ideal. Furthermore, knowing the fact that the strength and the orientation of a static electric field is a function of the distribution of the static electric charge makes the number of the designs limitless. For the sake of transparency and simplicity, in this article we consider a circular charged ring with a uniform positive charge distribution. The field along the axis through the center of the ring and perpendicular to the plane of the ring sustains its direction; it orients itself along the symmetry axis heading outward from the center. The strength of the field however, varies as a function of distance from the center; interestingly, its variation is not linear. Placing a negatively charged particle along a horizontal frictionless axis of the ring exerts a force on the particle accelerating it toward the center of the ring. The particle sling shots through the center of the ring and slides to the opposite 
side of it. On the other side of the ring because the field is reversed and reoriented, the particle decelerates and slides to a momentarily halt at a symmetrical opposite end. Since there is no loss of energy, continuous repetition of the movement is warranted and results in steady oscillations. The electric-spring in addition to being massless is nonlinear as well. Therefore, without idealizing the setup we are proposing a design resulting in perfect nonlinear oscillations. As mentioned earlier, in general, the shape of the nonlinear electric field is a function of shape of the configuration of the charge distribution. Hence, by replacing the ring with different geometrical shapes such as a square, a rectangle, an ellipse, an $\mathrm{n}$-tagon, and etc. one may fabricate limitless practical nonlinear electric-springs.

This article is composed of seven sections. Section 1 addresses the introduction and motivations, Section 2 outlines the objectives. Section 3 deals with the analysis. Section 4 addresses the energy issues of the oscillations, Section 5 embodies the 3D animation and the accompanied Mathematica code, Section 6 describes the extended design features and Section 7, the last section, shares the conclusions and closing remarks.

\section{Objectives}

We begin with a positively charged ring of radius R. Assuming the ring is made of a conductor; its charge distribution is uniform. Along the symmetry axis of the ring, the electric field, intuitively and justifiably is oriented along the axis heading outward from the center extending on both sides of the ring. The field along the axis due to the symmetry of the ring is distance dependent only. For any off-axis point the field depends on other coordinates as well. Apply the superposition principal to the electric field for a point along the symmetry axis trivially calculates [7]:

$$
E(x)=k Q \frac{x}{\left(R^{2}+x^{2}\right)^{\frac{3}{2}}}
$$

We have assumed a right handed Cartesian coordinate system, and the ring is placed in the yz plane with the $x$ axis stretching from left to right. The $x$ is the distance from the center of the ring, $Q$ is the charge on the ring and the value of $k=1 /\left(4 \pi \varepsilon_{0}\right) \equiv 9.0 \times 10^{9}$ in MKS units. A plot of $E /(k Q)$ for a $10 \mathrm{~cm}$ ring size is shown in Figure 1.

$$
\text { EfieldRing }\left[\mathrm{x}_{-}\right]=\frac{\mathrm{x}}{\left(\mathrm{R}^{2}+\mathrm{x}^{2}\right)^{\frac{3}{2}}} ;
$$

plotEfieldRing $=$ Plot $\left[\right.$ EfieldRing $[\mathrm{x}] / \mathrm{R} \rightarrow 10 \times 10^{-2},\{\mathrm{x},-1,1\}$, PlotRange $\rightarrow$ All,PlotStyle $\rightarrow\{$ Green,Thick $\}$, GridLines $\rightarrow$ Automatic, AxesLabel $\rightarrow\left\{\right.$ “x $(\mathrm{m})$ ",,'E/(k Q) $\left(1 / \mathrm{m}^{2}\right) "$ " $\left.\}\right]$

Equation (1) is an asymmetric/odd function with respect to $x$. Its plot displayed in Figure 1 shows the field for

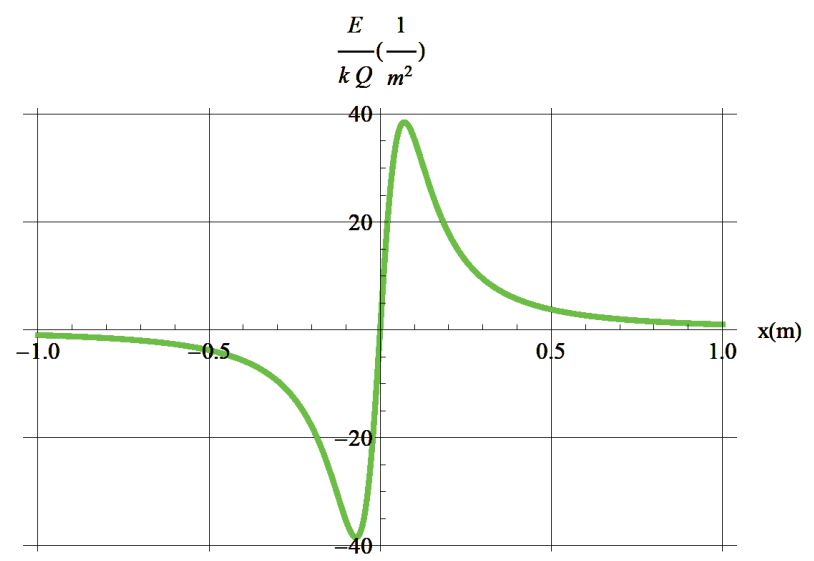

Figure 1. Display of $E /(k Q)$ for a ring of radius $R=10 \mathrm{~cm}$ along the symmetry axis vs. distance $x$ from the center of the ring

$\mathrm{x}<0$ is negative. This means the vector field $\vec{E}$ on both sides of the ring orients itself away from the center of the ring. The value of the electric field and its variation vs. distance indeed may be viewed as the characteristic of the electric force that would act on a charged particle if it were placed on the symmetry axis. Now we envision placing a negatively charged particle on the axis. Under the influence of such a nonlinear force the particle attractively accelerates toward the center of the ring. According to Figure 1, the electric field and therefore the electric force are entirely nonlinear. Its strength and magnitude varies quite differently vs. the strength of an idealized linear mechanical spring. For instance, one of the peculiar characteristics of the field as depicted in Figure 1 is its hump; at a certain distance from the center of the ring it becomes extremum. The abscissa of the latter is a function of the radius of the ring. These are:

$$
\begin{gathered}
\text { dEfieldRing }=\mathrm{D}[\text { EfieldRing }[\mathrm{x}], \mathrm{x}] / / \text { Simplify; } \\
\text { Solve[dEfieldRing }==0, \mathrm{x}] ;\left\{\left\{\mathrm{x} \rightarrow-\left(\frac{R}{\sqrt{2}}\right)\right\},\left\{\mathrm{x} \rightarrow \frac{R}{\sqrt{2}}\right\}\right\}
\end{gathered}
$$

Therefore, one objectively may select a ring size, $R$, to control the position along the symmetry axis where the field is extremum and its impact is the maximum. We now utilize the field; we place a particle with a chosen mass and charge $\{m, q\}$ along the symmetry axis of the ring. The opposite "sign" of the charged particle, in our case study, a negative charge, experiences an attractive force toward the center of the ring. The electric force accelerates the particle. While sliding, it experiences a position-dependent and a variable nonlinear force. Assuming a frictionless slide, the particle makes it to the center of the ring. With its velocity at the center it slings shots to the opposite side. While sliding on the opposite side of the ring and because of the reoriented field the particle experiences a retarding position-dependent force, 
decelerating it to a momentarily halt. The lack of friction preserves the energy of the particle, causing the movement to repeat itself endlessly. The particle therefore oscillates; however, because of the non-linearity of the imposed electric force the oscillation is nonlinear. The detailed analysis of the nonlinear oscillations along with related topics of interest follows.

\section{Analysis}

To form the equation describing the motion of the particle we begin with Newton's second law, $\vec{F}_{n e t}=m \vec{a}$. The particle is characterized with $\{m, q\}$. At any given time the electric force is the only effective force acting on the particle, $\vec{F}_{n e t} \equiv \vec{F}_{q}=q \vec{E}(x)$, where $E(x)$ is subject to Equation (1). Newton's law yields,

$$
\ddot{x}(t)+\frac{k Q q}{m} \frac{x(t)}{\left(R^{2}+x^{2}(t)\right)^{\frac{3}{2}}}=0
$$

The over-dot is defined as the derivative of the variable with respect to time, $t$. Equation (2) describes the motion of the particle. It is quite different from the classic equation describing the linear oscillations of a linear springmass system. It is a highly nonlinear differential equation. As we pointed out in the Introduction, historically, the Duffing equation is used as a pivotal equation describing the non-linear oscillations. A careful analysis of the force at hand reveals its masked correlation with the Duffing force. To unveil the correlation we expand the second term of Equation (2) about the center of the ring. Aside from the constant coefficient this gives:

$$
\begin{gathered}
\text { Series }\left[\frac{\mathrm{x}}{\left(\mathrm{R}^{2}+\mathrm{x}^{2}\right)^{\frac{3}{2}}},\{\mathrm{x}, 0,8\}\right] / / \text { PowerExpand } \\
\mathrm{x} / \mathrm{R}^{3}-\left(3 \mathrm{x}^{3}\right) /\left(2 \mathrm{R}^{5}\right)+\left(15 \mathrm{x}^{5}\right) /\left(8 \mathrm{R}^{7}\right)-\left(35 \mathrm{x}^{7}\right) /\left(16 \mathrm{R}^{9}\right)+\mathrm{O}[\mathrm{x}]^{9}
\end{gathered}
$$

This shows our proposed mass-less electric-spring is an upgraded description of the Duffing force. The first two terms of the expanded function is known to describe a "soft" spring [5]; and that limits the scope of the Duffing force. Simply put, the author's electric-spring is a superstructure version of the Duffing spring; i.e. its unexpanded function as it is used in Equation (2) embodies infinite odd powers of the compressed/elongated elec- tric spring's length; plus it describes an ideal/perfect mass- less entity.

Now we go back to Equation (2). At the outset, it is known that the Duffing equation given in the introduction has no analytic solution. For our superstructure case too, we were not surprised failing to solve Equation (2) analytically. We deploy Mathematica, but it also fails to solve symbolically. Lastly, we supply a set of practical initial conditions and by utilizing Mathematica we seek for a numeric solution. The code leading the numeric solution, along with the relevant computed kinematic quantities such as position, velocity and acceleration i.e. $\{x(t), v(t), a(t)\}$, respectively are given. The output is displayed. For the ring and the particle we assumed $\{R, Q\}=$ $\left\{10 . \times 10^{-2} m, 4.0 n C\right\}$, and $\{m, q\}=\{10 . m g, 3.0 n C\}$, respectively,

values $=\left\{\mathrm{k} \rightarrow 9.10^{9}, \mathrm{R} \rightarrow 10.10^{-2}, \mathrm{Q} \rightarrow 4.10^{-9}, \mathrm{~m} \rightarrow 10.10^{-6}\right.$, $\left.\mathrm{q} \rightarrow 3.10^{-9}\right\}$;

$$
\text { eqn }=x \text { " }[\mathrm{t}]+\left((\mathrm{k} Q \mathrm{q}) / \mathrm{m} \frac{\mathrm{x}[\mathrm{t}]}{\left(\mathrm{R}^{2}+\mathrm{x}[\mathrm{t}]^{2}\right)^{\frac{3}{2}}}\right) / . \text { values; }
$$

soleqn $=\mathrm{NDSolve}\left[\left\{\right.\right.$ eqn $==0, \mathrm{x}[0] 8(\mathrm{R} /$.values $), \mathrm{x}^{\prime}[0]==$ $0\}, \mathrm{x}[\mathrm{t}],\{\mathrm{t}, 0,50\}]$;

$\{$ position $x$, velocityx,acc $\}=\{x[t] /$.soleqn, $D[x[t] /$.soleqn, $\{\mathrm{t}, 1\}], \mathrm{D}[\mathrm{x}[\mathrm{t}] /$.soleqn, $\{\mathrm{t}, 2\}]\}$;

plotx $=$ Plot[positionx,$\{\mathrm{t}, 0,50\}$, AxesLabel $\rightarrow\{$ “t, s", , $\mathrm{x}, \mathrm{m}$ " $\}$, PlotStyle $\rightarrow$ Thick, GridLines- $>$ Automatic];plotv $=$ Plot[velocityx, $\{t, 0,50\}$, AxesLabel $\rightarrow\{$ “t,s", ,v,m/s" $\}$, PlotStyle $\rightarrow$ Thick, GridLines->Automatic];

plota $=$ Plot $\left[\right.$ accx,$\{t, 0,50\}$, AxesLabel $\rightarrow\left\{\right.$ "t $\left., \mathrm{s} ", " a, m / s^{2} "\right\}, P 1$ otStyle $\rightarrow$ Thick, GridLines->Automatic];

Show[GraphicsArray[\{plotx,plotv,plota\}],ImageSize $\rightarrow$ 500]

The far left graph of Figure 2 indeed confirms our speculated intuitive expected oscillations.

The particle oscillates about the origin, i.e. the center of the ring. A trained and experienced eye clearly would be able to distinguish the difference between the characteristic of this nonlinear oscillations vs. linear classic simple harmonic oscillations. From this plot alone one may deduce useful information such as period, which for the chosen utilized parameters is $32.0 \mathrm{~s}$. The initial distance of the particle from the center of the ring is $8 R=0.8 \mathrm{~m}$. It takes $8.0 \mathrm{~s}$ for the particle to make it to the center. At that
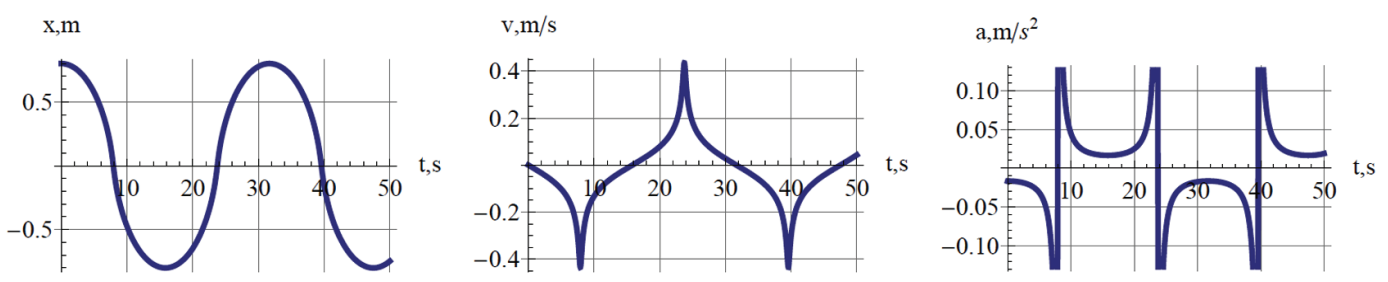

Figure 2. Display of the position $x$, velocity $v$ and acceleration a of the charged particle vs. $t$, respectively from left to right 

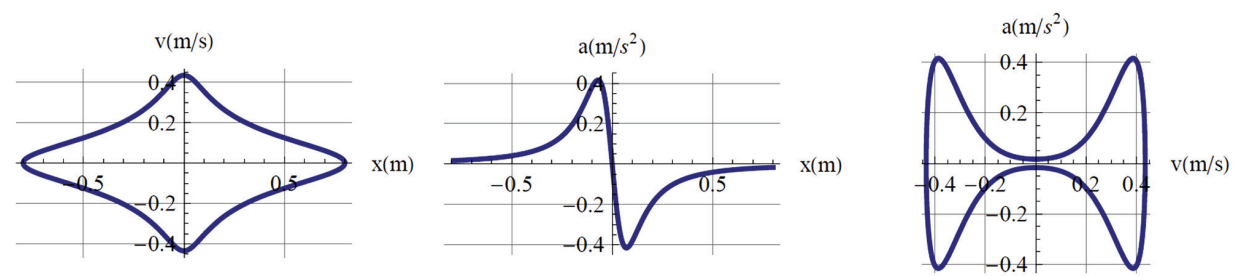

Figure 3. Plots of $\{x(t), v(t)\},\{x(t), a(t)\}$ and $\{v(t), a(t)\}$ from left to right, respectively

instance, according to the center graph of Figure 2, the velocity of the particle is at maximum; its acceleration according to the far right graph of Figure 2 is at maximum. From this instant moving forward, the particle continues sliding to the left, while continually loosing speed. When the clock ticks $16.0 \mathrm{~s}$ its instantaneous speed plunges to zero; at that moment it reverses its motion speeds up, and accelerates toward the center of the ring.

To display the differences between the characteristics of the classic simple harmonic motion vs. our designed nonlinear oscillations, we display three phase diagrams. Utilizing Mathematica ParametricPlot we fold the time parameter and plot a set of three kinematic pairs: $\{x(t), v(t)\},\{x(t), a(t)\}$ and $\{v(t), a(t)\}$.

plotxv $=$ ParametricPlot[Flatten[ \{positionx,velocityx $\}],\{\mathrm{t}$, $0,50\}$,AxesLabel $\rightarrow\{$ “ $\mathrm{x}(\mathrm{m})$ ", " $\mathrm{v}(\mathrm{m} / \mathrm{s})$ " $\}$, PlotStyle $\rightarrow$ Thick, GridLines $\rightarrow$ Automatic,PlotRange $\rightarrow$ All];

plotxa $=$ ParametricPlot[Flatten[\{positionx,accx $\}],\{\mathrm{t}, 0,50\}$ ,AxesLabel $\rightarrow\left\{\right.$ “" $\mathrm{x}(\mathrm{m})$ ", "“a $\left(\mathrm{m} / \mathrm{s}^{2}\right)$ " $\}$, PlotStyle $\rightarrow$ Thick, Grid Lines $\rightarrow$ Automatic,PlotRange $\rightarrow$ All];

plotva $=$ ParametricPlot[Flatten[\{velocityx, accx $\}],\{t, 0,50\}$ ,AxesLabel $\rightarrow\left\{\right.$ “v $(\mathrm{m} / \mathrm{s})$ ", “a $\mathrm{a}\left(\mathrm{m} / \mathrm{s}^{2}\right)$ " $\}$, PlotStyle $\rightarrow$ Thick,Gri dLines $\rightarrow$ Automatic,PlotRange $\rightarrow$ All];

Show[GraphicsArray[\{plotxv,plotxa,plotva\}],ImageSize $\rightarrow 500]$

As one may speculate, the shapes displayed in Figure 3 are sensitive to the chosen parameters $\{R, Q\}$ and $\{m, q\}$. The interested reader may run the code for various parameters.

\section{Energy of the Nonlinear Oscillator}

In the analysis of the classic linear harmonic oscillations one displays the interplay of the kinetic and potential energies of the oscillator vs. time, $t$. For a conservative setting i.e. when the friction forces are ignored, the sum of these two energies over the span of the oscillations remains constant. Utilizing the same assumption, a nonlinear oscillator preserves its energy as well; however, the time dependent variation of its kinetic and the potential energies are quite different from the linear counter case. We utilize the solution of Equation (2). The potential and kinetic energies of the oscillators are: $\mathrm{PE}=\frac{k Q q}{\sqrt{R^{2}+x(t)^{2}}}$ and $\mathrm{KE}=1 / 2 m v(t)^{2}$, respectively.

$\{\mathrm{PE}, \mathrm{KE}$, totalEnergy $\}=\left\{-\mathrm{kQq} \frac{1}{\sqrt{\mathrm{R}^{2}+\text { positionx }^{2}}}, 1 / 2 \mathrm{~m}\right.$

velocityx $x^{2},\left(1 / 2 \mathrm{~m}\right.$ velocityx $\left.\left.{ }^{2}-\mathrm{kQq} \frac{1}{\sqrt{\mathrm{R}^{2}+\text { position }^{2}}}\right)\right\}$

//.values;

Plot $\left[\left\{10^{7} \mathrm{PE}, 10^{7} \mathrm{KE}, 10^{7}\right.\right.$ totalEnergy $\},\{\mathrm{t}, 0,20\}$, PlotStyle $\rightarrow\{\{$ Thick, GrayLevel[0.5]\},\{Thick,Black $\},\{$ Thick,Dashi $\mathrm{ng}[\{0.02\}]$, GrayLevel $[0.2]\}\}$,PlotRange $\rightarrow$ All,AxesLabel $\rightarrow\{$ "t,s", "Energy,J" $\}$, GridLines Automatic]

For the sake of displaying the energies with a meaningful scale the energies are magnified by a factor of $10^{7}$. According to Figure 4 and as we discussed in the previous section, the oscillator with an initial zero speed begins sliding toward the center of the ring. Since it is farthest from the ring, its potential and kinetic energies are at minimum and zero values, respectively. These are shown by the far left tails of the curves in Figure 4 . While the oscillator accelerates toward the ring, its potential energy decreases and the speeding particle gains kinetic energy. When the oscillator is at the center of the ring, its potential energy is at minimum; conversely its kinetic energy is at maximum. At any given instance the sum of the values of the potential and the kinetic energies stays constant; the dashed horizontal line confirms the fact.

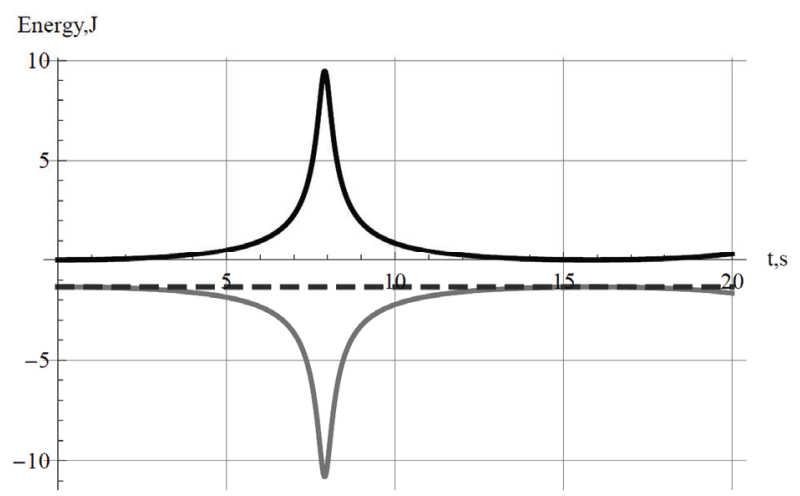

Figure 4. Display of the potential energy (the light gray curve), kinetic energy (the black curve) and the total energy (the dashed line) vs. time 


\section{3D Real-Life Animation and the Art Work}

A sinusoidal time dependent function describes the oscillations of a classic linear oscillator. As an example we may envision the swinging movement of a simple pendulum under gravity's pulls. How does a nonlinear oscillator swing? Applying the solution of Equation (2) and utilizing Mathematica's animation feature we compose a code to bring the nonlinear oscillations to life. The animation depicts a positively charged ring in red, a negatively charged particle in blue, and the electric field/force in green. The released particle from its initial positive position on the $\mathrm{x}$-axis moves toward the ring. For the chosen ring size, the maximum value of the field/force occurs in the vicinity of its center; at $x=\frac{R}{\sqrt{2}}$. The animation clearly shows the impact of the nonlinear field. i.e., when the particle makes it to the maximum field, the field jerks the particle and radically changes its movement.

plot3d $1=$ ParametricPlot3D $\left[\left\{0, \mathrm{x}, \sqrt{1-\mathrm{x}^{2}}\right\},\{\mathrm{x},-1,1\}\right.$,PlotStyle $\rightarrow\{$ Red,Thick $\}$,PlotRange $\rightarrow\{-1,1\}$,AxesLabel $\rightarrow$ $\{$ ‘x","y","z"\}];

plot3d2=ParametricPlot $3 \mathrm{D}\left[\left\{0, \mathrm{x},-\sqrt{1-\mathrm{x}^{2}}\right\},\{\mathrm{x},-1,1\}\right.$,PlotStyle $\rightarrow\{$ Red,Thick $\}$, PlotRange $\rightarrow\{\{-1,1\},\{-1,1\},\{-1,1\}\}$, AxesLabel $\rightarrow\{$ “x","y","z"\}];

Show [ \{plot3d1,plot3d2\}]; plotlinex $=$ ParametricPlot $3 \mathrm{D}[\{\mathrm{t}, 0,0\},\{\mathrm{t},-1,1\}$, PlotRange $\rightarrow\{\{-1,1\},\{-1,1\},\{-1,1\}\}$, PlotStyle $\rightarrow$ Thick $]$; plotliney $=$ ParametricPlot3D $[\{0, \mathrm{t}, 0\},\{\mathrm{t},-1,1\}$, PlotRange $\rightarrow\{\{-1,1\},\{-1,1\},\{-1,1\}\}$, PlotStyle $\rightarrow\{$ Dashing[0.02],Thi $\mathrm{ck}\}]$; plotlinez $=$ ParametricPlot $3 \mathrm{D}[\{0,0, \mathrm{t}\},\{\mathrm{t},-1,1\}$, PlotRange $\rightarrow\{\{-1,1\},\{-1,1\},\{-1,1\}\}$, PlotStyle $\rightarrow\{$ Dashing[0.02],Thi ck\}];

Show [\{plot3d1,plot3d2,plotlinex,plotliney,plotlinez $\}$; EfieldRing3D $=$ ParametricPlot3D $[\{\mathrm{x}, 0,1 / 40$ Evaluate[EfieldRing $[\mathrm{x}] /$.values $]\},\{\mathrm{x},-1,1\}$, PlotStyle $\rightarrow\left\{\right.$ Green $\left({ }^{*}\right.$ GrayLevel[0.5]*),Thick $\}$;

Manipulate[Show[\{plot3d1,plot3d2,plotlinex,plotliney, plotlinez,Graphics3D[\{Blue,Sphere[\{positionx[[1]]/.t $\rightarrow$ $\tau, 0,0\}, 0.05]\}]$,EfieldRing3D $\},$ FaceGrids $\rightarrow\{\{0,0,1\},\{0,0$, $-1\}\}$,FaceGridsStyle $\rightarrow$ Directive[Black,Dashed,Thickn$\operatorname{ess}[0.003]]],\{\tau, 0,50,0.1\}]$

DynamicModule $[\{\tau=0\}$, Show [ \{plot3d1,plot3d2,plotlinex,plotliney,plotlinez,Graphics3D[\{Blue,Sphere[\{positio$\left.\left.\left.\mathrm{nx}[[1]] / . \mathrm{t} \rightarrow \tau, 0,0\}, 0.05^{\circ}\right]\right\}\right]$, EfieldRing3D $\}$, FaceGrids $\rightarrow\{$ $\{0,0,1\},\{0,0,-1\}\}$,FaceGridsStyle $\rightarrow$ Directive[Black,Dashed,Thickness[0.003']]]]

\section{Extended Design Possibilities}

Utilizing the concepts described in the previous section, one may readily fabricate limitless electric-springs. Without deviating too far from what we have already described, we introduce a charged double-ring electric-spring. For instance, by adjusting the values of the charge densi-

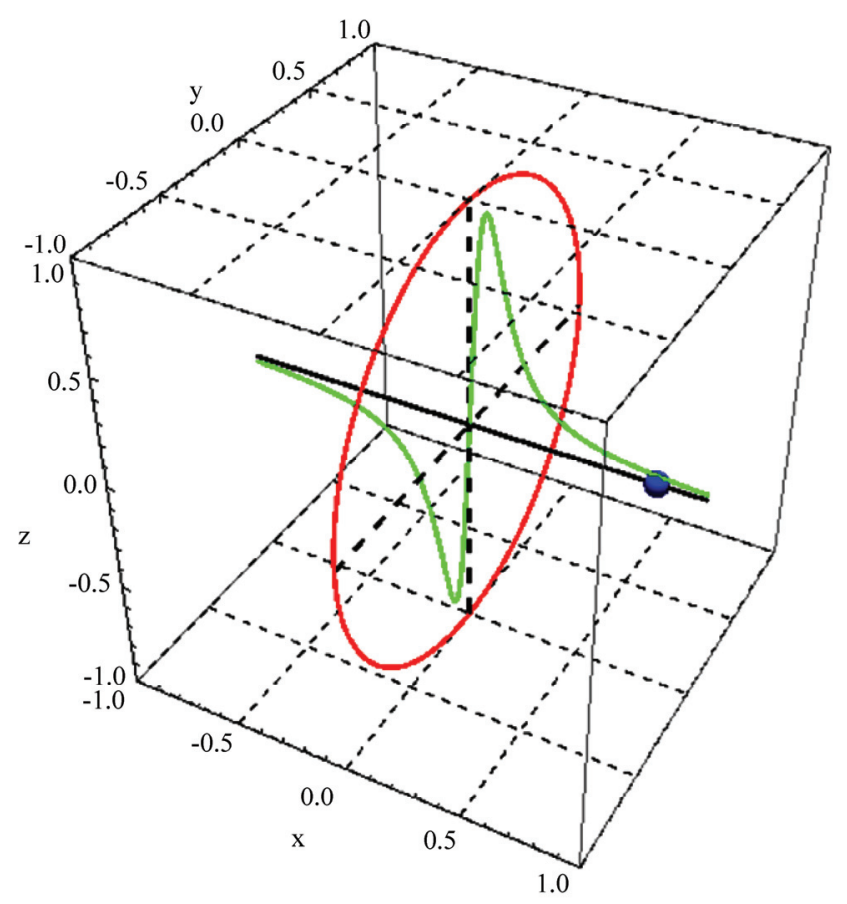

Figure 5. A snapshot of animated movement of a negatively charged particle (the black dot), within the electric field (the light grey signal) of a positively charged ring (the black circle) 


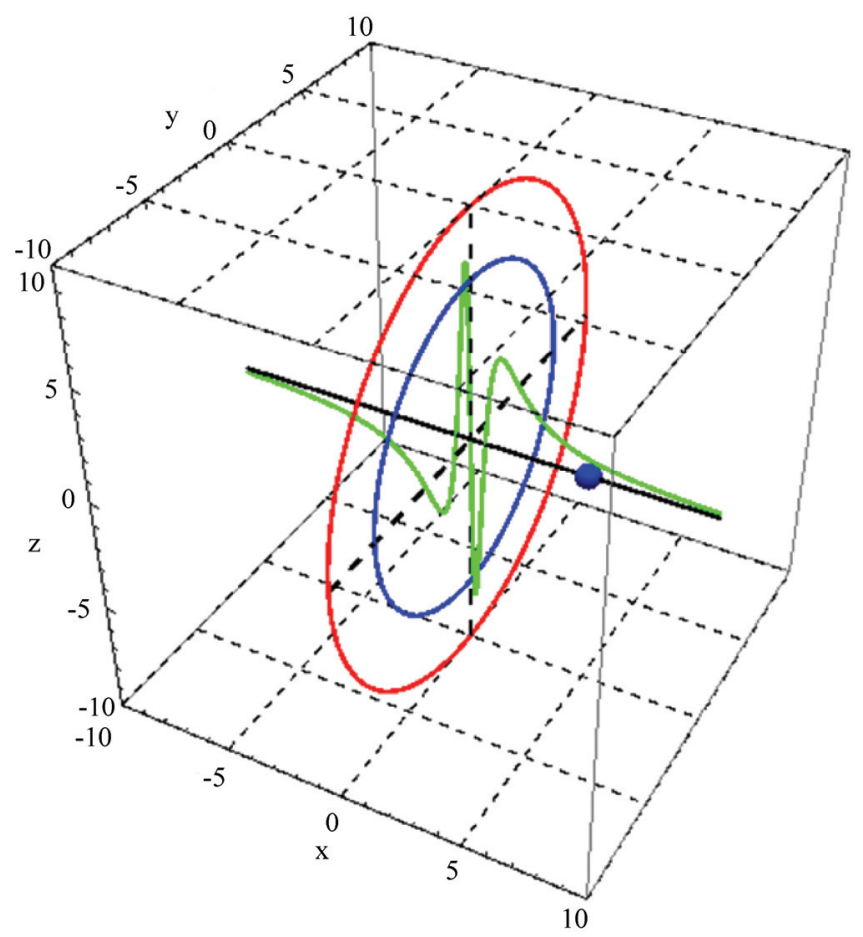

Figure 6. A snapshot of the motion of the negatively charged particle (the black ball), and the double-ring. The outer ring is positively charged, the inner ring is negatively charged, and each ring has its own linear charge densities. The electric field along the common ring axis is in light grey
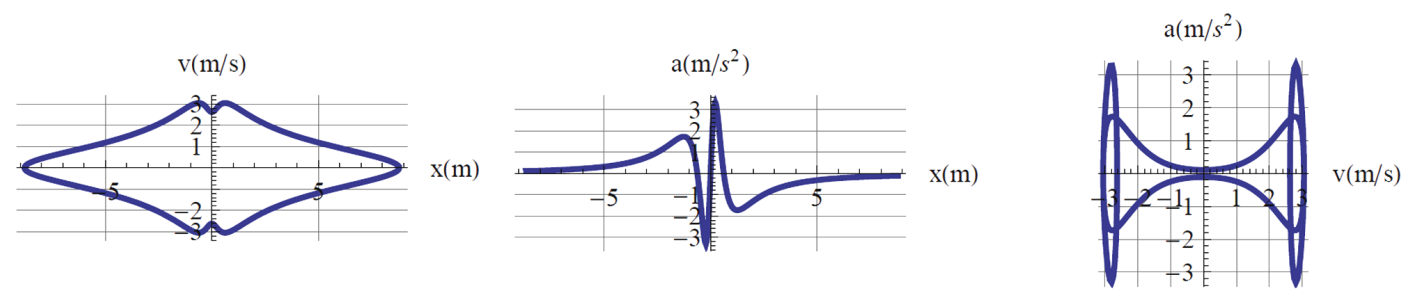

Figure 7. Plots of $\{x(t), v(t)\},\{x(t), a(t)\}$ and $\{v(t), a(t)\}$ are displayed in left, center and right diagrams, for the charged double-ring, respectively

ties of the rings, e.g. a positive density for the outer ring and a negative density for the inner ring, we were able to produce a double hump electric field. In Figure 6, the field is depicted in green. The positions of the humps are a function of the rings' radii. By choosing a set of appropriate radii one may set the abscissa of the humps at desired positions. The field of the double-ring electric- spring shown in Figure 6 is distinctly different from the one shown in Figure 1. Utilizing the former we form the equation describing the movement of a negatively charged particle. The equation of motion is similar to Equation (2). However, because of the shape of the electric field the solution of the equation and therefore the description of the motion is distinctly different from what we have shown in the previous section. We compose a Mathematica code and animate the motion. This is shown in Figure 6.
Applying the solution of the equation of motion we plot three phase diagrams similar to the ones displayed in Figure 3. These are shown in Figure 7.

Comparing Figure 7 with Figure 3, one clearly sees the drastic differences. The impact of the double-ring on the oscillations of the oscillator is significant. The most notable impacts are displayed in the far left and the far right plots of Figure 7.

\section{Conclusions and Remarks}

In this article the author introduces a perfect mass-less static electric-spring design resulting nonlinear mechanical oscillations. The core idea of the design is based on the configuration of the electric field of a suitable static charge distribution. Utilizing the symmetry of a circular ring, it is shown that the field along the axis of the ring 
has the desired characteristic. The magnitude of the field along the axis varies non-linearly with distance making the force that acts on the particle nonlinear as well. It is also shown how the design is modified. For instance the details of a coplanar charged double-ring is discussed and exhibits an interesting non-linearity behavior. A thorough literature search reveals no such practical designs have been proposed earlier. Furthermore, based on the aforementioned detailed discussions one realizes that the number of blue print electric-spring designs are limitless. Therefore, one may design springs to meet any practical needs. The available literature on the mechanical nonlinear physics phenomenon mostly is discussed from a purely theoretical view. In this article we have shown practical and applicable applications. We also would like to point out that in our study because of the slowness of the motion of the charged particle justifiably we ignored the effects of electromagnetic radiation of the oscillator.

\section{REFERENCES}

[1] S. Wolfram, "The Mathematica book," 5th Ed., Cambridge University Publications, 2003.

[2] E.g. J. Marion, "Classical dynamics of particles and systems," 4th Ed., Harcourt College Publishers, 1995.

[3] G. Duffing, "Erzwungene schwingungen bei veranderlicher eigen-frequenz," F. Vieweg und Sohn, Braunschweig, 1918.

[4] S. Strogatz, "Nonlinear dynamics and chaos," Perseus Publishing, 1994.

[5] http://wow.scalped.orgy/article/Duffing_oscillator.

[6] R. H. Ennis and G. C. McGuire, "Nonlinear physics with Mathematica for scientists and engineers," Published by Birdhouse, Hard Spring, Peg 605, 2001.

[7] E.g. J. D. Jackson, "Classical electrodynamics," 3rd Ed., Wiley, 2005. 